\title{
Oportunidades, percalços e justificativas: a descentralização da regulação ambulatorial no município do Rio de Janeiro
}

\author{
Opportunities, drawbacks and justifications: outpatient care \\ regulation's decentralization in the city of Rio de Janeiro
}

Kennedy Guabiraba1', Gustavo Gomes², Eduardo Alves Melo $\mathbf{1 , 2}$

DOI: $10.1590 / 0103-1104202213207$

RESUMO Desde 2012, o município do Rio de Janeiro possui a inovação no cenário nacional de ter a regulação do acesso à atenção especializada feita por médicos na Atenção Primária à Saúde (APS). Este artigo analisa o processo técnico-político que produziu a descentralização da regulação ambulatorial para APS: suas motivações e contexto, atores, apostas, decisões e desdobramentos. Produzido através de entrevistas com gestores, analisou-se o material tendo como referência principal o ciclo de políticas públicas de Howlett e Ramesh, de modo que suas etapas inspiraram a categorização adotada no artigo. O movimento de descentralização da regulação ambulatorial apoiou-se em insatisfações com o acesso ambulatorial. Em contexto de fortalecimento da APS, disputa pelo controle de recursos assistenciais e utilizando a coordenação do cuidado como elemento discursivo, o modelo viabilizou maior protagonismo de APS na regulação do acesso. Em contrapartida, alguns ‘sucessos' não são reflexos exclusivos da descentralização para a APS, e surgem consequências negativas. A partir da adoção de processos avaliativos, pode-se viabilizar a criação de outros arranjos regulatórios num modelo que permanece promissor.

PALAVRAS-CHAVE Acesso aos serviços de saúde. Regulação e fiscalização em saúde. Atenção Primária à Saúde. Política de saúde.

1 Universidade Federal Fluminense (UFF) Niterói (RJ), Brasil. kennedyguabiraba@gmail. com

2 Fundação Oswaldo Cruz (Fiocruz) - Rio de Janeiro (RJ), Brasil.
ABSTRACT Since 2012, the city of Rio de Janeiro has the innovation on the national scene of having the regulation of access to specialized care made by physicians in Primary Health Care (PHC). This article analyses the technical-political process that produced outpatient regulation decentralization to $P H C$ : its motivations and context, actors, stakes, decisions and ramifications. Produced from interviews with managers, the material was analyzed having as main reference the public policies' cycle of Howllet and Ramesh, so that its steps inspired the categories adopted in the article. The movement to decentralize outpatient regulation was based on dissatisfaction with outpatient access. In a context of strengthening the PHC, disputes for the control of care resources and using the care coordination as a discursive element, the model enabled greater role for $\mathrm{PHC}$ in access' regulation. Nonetheless, some 'successes' are not exclusive reflections of decentralization for $\mathrm{PHC}$, and negative consequences arise. From the adoption of evaluation processes, it is possible to create other regulatory arrangements in a model that remains promising.

KEYWORDS Health services accessibility. Health care coordination and monitoring. Primary Health Care. Health policy. 


\section{Introdução}

Organizar os sistemas de saúde é importante desafio para a construção de cuidados integrados, de modo que a regulação surge, no Brasil, para gerenciar o funcionamento e a relação dos pontos de atenção, como formas de entrada e encaminhamentos $\mathbf{1}^{\mathbf{1}} \mathbf{2}$. Das modalidades regulatórias, destaca-se a regulação assistencial (ou do acesso), nos âmbitos ambulatorial, hospitalar e de urgência/emergência, e que:

Tem como objetos a organização, o controle, o gerenciamento e a priorização do acesso e dos fluxos assistenciais no âmbito do Sistema Único de Saúde (SUS), e como sujeitos seus respectivos gestores públicos, sendo estabelecida pelo complexo regulador e suas unidades operacionais e esta dimensão abrange a regulação médica, exercendo autoridade sanitária para a garantia do acesso baseada em protocolos, classificação de risco e demais critérios de priorização ${ }^{3}$.

Essa regulação em redes de atenção, também entendida como gestão das listas de espera em diversos países com sistemas universais de saúde ${ }^{4}$, é considerada estratégica para acesso aos cuidados de modo equânime, adequado e em tempo oportuno. Ainda assim, a regulação ambulatorial (acesso a consultas, exames ou procedimentos especializados) aparece muitas vezes como um 'gargalo' no fluxo de usuários pelo Sistema Único de Saúde (SUS) 5 . Além disso, tensões entre os regimes clientelístico e governamental são frequentes em experiências que buscam tornar mais transparente o controle dos recursos assistenciais especializados e mais equânime o acesso ${ }^{6}$.

A Atenção Primária à Saúde (APS), por sua vez, como coordenadora do cuidado nas redes de atenção $0^{\mathbf{2}, \mathbf{8}}$, é fundamental para a transição adequada aos cuidados de nível especializado, e diversos estudos nacionais têm apontado que sua participação ainda é tímida, embora frequente, na regulação do acesso. Nesse contexto, algumas experiências e estudos têm abordado a participação da APS no processo de regulação, incluindo sua interface com a atenção especializada ${ }^{9-12}$.

Desde 2012, o município do Rio de Janeiro (MRJ), capital do estado, tem a maioria dos processos referentes à regulação ambulatorial acontecendo na APS, pois médicos das unidades básicas passaram a realizar atividades como avaliação, autorização, agendamento, devolução ou negação de solicitações feitas por colegas num mesmo serviço ${ }^{13}$. Esse modelo, chamado descentralizado, concebido em um contexto de fortes investimentos municipais na APS que perduraram até o momento da troca de gestão em 2017, distribuiu parte das atribuições regulatórias, antes exclusivas da central municipal de regulação, para a APS, e ainda não foi alvo de muitos estudos sobre seu processo de construção, funcionamento e organização ${ }^{13-15}$.

Este artigo tem como objetivo analisar o processo técnico-político que produziu a descentralização da regulação ambulatorial para a APS no MRJ, notadamente, suas motivações e contexto, atores, apostas, decisões e desdobramentos.

\section{Metodologia}

Trata-se de um estudo qualitativo, representando um recorte do projeto de pesquisa 'A regulação do Acesso à Atenção Especializada na Atenção Primária à Saúde'. O MRJ, cenário do estudo, possui cerca de 6.718.903 habitantes ${ }^{16}$, sendo dividido pela Secretaria Municipal de Saúde (SMS-RJ) em 10 áreas programáticas ${ }^{17}$, embora exista entre elas heterogeneidade de serviços e população ${ }^{13}$. O quadro 1 caracteriza as atenções primária e especializada da cidade. 
Quadro 1. Caracterização de organização, serviços e gestão de APS e atenção especializada do município do Rio de Janeiro - Rio de Janeiro, 2021

\begin{tabular}{|c|c|c|c|}
\hline & Organização & Ações e serviços & Gestão \\
\hline $\begin{array}{l}\text { Atenção pri- } \\
\text { mária }\end{array}$ & $\begin{array}{l}\text { Majoritariamente formado } \\
\text { por unidades de APS no } \\
\text { modelo eSF, havendo uma } \\
\text { minoria que funciona no } \\
\text { modelo tradicional. Algu- } \\
\text { mas unidades possuem } \\
\text { especialidades e exames } \\
\text { ambulatoriais. }\end{array}$ & $\begin{array}{l}\text { Consultas em APS, pe- } \\
\text { quenos procedimentos } \\
\text { ambulatoriais, promoção } \\
\text { à saúde, vacinação, visitas } \\
\text { domiciliares e saúde bucal. }\end{array}$ & $\begin{array}{l}\text { As unidades se encontram sob gestão } \\
\text { municipal e são gerenciadas por meio } \\
\text { de organizações sociais de saúde. Res- } \\
\text { pondem diretamente ao nível central e } \\
\text { às coordenadorias regionais. }\end{array}$ \\
\hline $\begin{array}{l}\text { Atenção espe- } \\
\text { cializada }\end{array}$ & $\begin{array}{l}\text { Os espaços que integram } \\
\text { o nível secundário incluem } \\
\text { Policlínicas, Centros de } \\
\text { Especialidades Odontológi- } \\
\text { cas, Centros Especializados } \\
\text { de Reabilitação e ambu- } \\
\text { latórios hospitalares, por } \\
\text { exemplo. }\end{array}$ & $\begin{array}{l}\text { Exames (exames de } \\
\text { imagem, endoscopias e } \\
\text { biópsias) e consultas da } \\
\text { atenção especializada } \\
\text { (especialidades médicas, } \\
\text { odontológicas, em saúde } \\
\text { mental, fisioterápicas, } \\
\text { fonoaudiológicas). }\end{array}$ & $\begin{array}{l}\text { Entre os procedimentos ambulatoriais } \\
\text { (exames e consultas) sob regulação } \\
\text { municipal, estima-se que } 60,1 \% \text { são } \\
\text { municipais, } 24,2 \% \text { são de prestadores } \\
\text { privados, } 7,4 \% \text { são de hospitais e insti- } \\
\text { tutos federais contratados e } 8,3 \% \text { são } \\
\text { de hospitais universitários. Boa parte } \\
\text { dos serviços especializados sediados } \\
\text { na cidade do Rio de Janeiro é acessada, } \\
\text { também, por usuários de outras cidades } \\
\text { do estado, a partir de pactuações inter- } \\
\text { gestores. }\end{array}$ \\
\hline
\end{tabular}

Fonte: elaboração própria.

Para o presente artigo, realizaram-se entrevistas com 8 gestores da Secretaria Municipal de Saúde (SMS-RJ) - 4 centrais e 4 de área programática - ligados à regulação ambulatorial e à APS, tendo a maioria participado da construção do modelo de regulação ambulatorial descentralizada na gestão de 2009-2016, e outros ingressando a partir de 2017, após a implantação do modelo. As entrevistas, feitas entre julho/2019 e janeiro/2020, duraram, em média, uma hora, foram audiogravadas e, posteriormente, transcritas. Fez-se análise de conteúdo ${ }^{\mathbf{1 8}}$ do material, na sua modalidade temática. Fragmentos ou menções aos gestores entrevistados estão identificados no texto pelos nomes indicados no quadro 2.

Quadro 2. Gestores centrais e regionais entrevistados segundo gênero, profissão, área ou função de gestão e tempo na gestão. Rio de Janeiro, 2021

\begin{tabular}{llllcl}
\hline & Gênero & Profissão & Área ou função de gestão & $\begin{array}{c}\text { Tempo aproximado } \\
\text { na função }\end{array}$ \\
\hline Gestor 1 & Masculino & Médico & Superintendência de Atenção Primária (SMS-RJ)* & 3 anos \\
Gestor 2 & Feminino & Médica & Coordenação de Área Programática & 11 meses \\
Gestor 3 & Feminino & Médica & Coordenação de Regulação Ambulatorial (SMS-RJ) & 2 anos \\
Gestor 4 & Masculino & Médico & Alta direção da SMS-RJ & 2 anos \\
Gestor 5 & Feminino & Médica & Coordenação de Núcleo Interno de Regulação de Coorde- & 1 ano e 10 meses \\
& & & nadoria de Área Programática (CAP)* & 5 anos \\
Gestor 6 & Feminino & Médica & Coordenação de Regulação Ambulatorial (SMS-RJ)* & 1 ano \\
Gestor 7 & Feminino & Enfermeira & Coordenação de Área Programática* & 11 anos
\end{tabular}

Fonte: elaboração própria.

* Entrevistados que ocupavam função de gestão no momento da entrevista. 
Entende-se que o processo de construção da regulação descentralizada pode ser considerado uma resposta do governo (municipal, nesse caso) para um problema social, a partir da negociação de interesses e avaliações técnicas ${ }^{19}$, ou seja, como parte de uma política pública. Por isso, ele foi estudado a partir de categorias inspiradas no ciclo para análise de políticas públicas de Howlett e Ramesh, usado para examinar uma política pública a partir da complexa interação entre os atores e condições envolvidas no processo. As categorias originais do ciclo que inspiraram o presente trabalho são: montagem da agenda; formulação da política; tomada de decisão; implementação; avaliação ${ }^{20}$. Em cada categoria recorreu-se, também, a outras produções acadêmicas para a discussão dos seus resultados específicos.

A pesquisa foi aprovada por Comitês de Ética em Pesquisa da instituição dos pesquisadores e da SMS-RJ, sob os pareceres de número 3.263 .136 e 3.358.407, respectivamente. Os participantes foram esclarecidos dos objetivos da pesquisa e consentiram participar.

\section{Resultados e discussão}

\section{Montagem de agenda: descentralização no contexto de dificuldades de acesso ambulatorial}

O desenvolvimento de uma política, na perspectiva do ciclo de Howlett e Ramesh, inicia-se pelo reconhecimento do problema. Uma combinação entre fluxos de problemas, fluxos de soluções e fluxos políticos converge em janelas de oportunidades para criar ou modificar a agenda política pré-existente ${ }^{19}$.

$\mathrm{Na}$ entrada de novos temas na lista decisória do governo, destaca-se a participação de 'empreendedores de políticas', atores que advogam por propostas e investem recursos em prol de seus interesses, tornando-se protagonistas nos momentos de confluência das condições favoráveis a alterações nas agendas governamentais ${ }^{19,21}$.
O processo de descentralização da regulação no Rio de Janeiro se inicia em contexto de expansão da APS no município - até então, havia baixa cobertura pela Estratégia Saúde da Família ${ }^{13}$, além de predomínio das unidades com modelo chamado de tradicional. Nesse cenário, fortaleceram-se debates e movimentos relacionados ao acesso à atenção especializada.

Intensificou-se, por exemplo, a negociação pelo controle da oferta ambulatorial ${ }^{13}$. Aparentemente, disputas políticas confrontavam a tentativa de aumento do número de vagas no sistema de regulação da cidade frente à resistência de alguns prestadores da atenção especializada municipais, estaduais, federais e privados. Para gestores, pouco se justifica a tentativa dos prestadores de não disponibilizarem o pleno controle dos serviços para a central de regulação.

Uma grande resistência das pessoas de mostrarem qual é a sua vaga, quanto que você tem de vaga. [...] Não se tinha de forma alguma o total de vagas disponíveis para agendamento. (Gestor 3).

De acordo com alguns entrevistados, outro desafio para a SMS-RJ era o acesso informal às vagas na atenção especializada. Esses fluxos eram promovidos principalmente por forças anti ou contrarregulatórias, como atores locais que utilizavam a saúde para projetos político-partidário-eleitorais. Além disso, debatia-se a histórica fragmentação das informações relacionadas à regulação, pois muitos dos serviços estavam instalados na atenção terciária e eram gerenciados nesses locais independentemente da rede. Consequentemente, agendamentos no sistema de saúde municipal funcionavam em várias filas com fluxos divergentes e solicitações duplicadas.

Problemas de ordenação do cuidado ambulatorial eram cotidianos, contribuindo para um cenário em que, frequentemente, o usuário peregrinava entre prestadores para agendamento de procedimentos e consultas, tornando-se responsável por obter cuidado. Esse processo era árduo para o paciente e contribuía para 
uma realidade de filas extensas, iniciadas de madrugada nos locais prestadores. Ademais, os encaminhamentos não garantiam acesso ao especialista ou ao exame, tornando a saga mais sofrida.

Então, o paciente chegava. Se ele precisasse, por exemplo, de uma consulta de cardiologia na unidade, preenchia uma guia que chamava 'pedido de consulta de especialidade'. Dava para o paciente onde tinha a consulta e o paciente ia atrás. (Gestor 3).

A dificuldade crônica de acesso ambulatorial municipal diminuiu com esforços iniciados em 2009, que levaram a um crescimento da oferta de vagas sob regulação municipal, assim como a menor fragmentação e maior transparência das informações sobre encaminhamentos. Entretanto, vagas ambulatoriais sob controle municipal eram reguladas quase exclusivamente na central municipal de regulação, onde apenas 10 médicos eram responsáveis por todas as solicitações do MRJ14.

Logo, antes do modelo descentralizado, o cotidiano de trabalho na central de regulação era marcado por disparidade entre o volume analisado/regulado e a demanda de solicitações. Existia grande dificuldade para se obter o agendamento de consultas e procedimentos, além de sobra/perda de vagas ${ }^{15}$.

A central de regulação não tem funcionários para regular para todo o Rio de Janeiro, então demorava muito para que os pacientes conseguissem uma vaga. O prestadorpodia fornecer a vaga, mas eles não tinham como dar conta. (Gestor 7).

[...] tinha 5 ou 6 médicos para a cidade inteira, para fazer essas autorizações. (Gestor 1).

Essa realidade se configurava, inclusive, como irregularidade: seriam necessários 8 médicos reguladores com carga-horária de 20 horas semanais e 21 plantonistas de 12 horas para se cumprir o volume de trabalho de pelo menos $30 \%$ da oferta das primeiras consultas especializadas e $50 \%$ da oferta de procedimentos ambulatoriais de alta complexidade, conforme normativas federais $\mathbf{1 5}^{\mathbf{1 5 2}}$. Ademais, mesmo com a expansão do nível primário, as atividades da regulação não eram cotidianamente centrais na atenção básica, e esse espaço surgia na rede apenas como um 'consumidor' de vagas a partir de encaminhamentos.

Desse modo, apostou-se em aumentar a participação da APS na regulação em contexto de descontentamentos de usuários e profissionais (fluxo de problemas), somados a grande investimento municipal na expansão estrutural, organizacional e simbólica da APS, que despontava como protagonista na rede (fluxo de soluções). A aposta é parte de um movimento que se tornou mais robusto em 2009 (fluxo político) sob o protagonismo de atores/gestores relacionados às mudanças na APS carioca (empreendedores de políticas). Desse modo, o início do movimento descentralizador do MRJ exemplifica os fluxos descritos por Kingdon ${ }^{21}$, convergindo para criar a janela de oportunidade da mudança na regulação ambulatorial.

\section{Formulação da política: os discursos e elaborações que sustentaram a mudança}

A criação das soluções e alternativas é marcada por grande diálogo de intenções e ações, em que se busca estabelecer alternativas para a solução da problemática original, conciliando os diversos interesses dos atores envolvidos, as condições socioeconômicas e as regras político-institucionais dos processos atrelados às propostas formuladas ${ }^{19}$.

A partir dos debates iniciados na etapa anterior, e alinhada com outros movimentos da política de expansão da APS, a regulação descentralizada passa a ser proposta por gestores municipais para aperfeiçoar a coordenação do cuidado pela APS. Segundo entrevistados, a descentralização seria capaz, também, de aproximar médicos das realidades dos territórios e, ao mesmo tempo, aumentar 
a capacidade regulatória tanto da unidade quanto do município, a fim de organizar a rede para romper com o processo prévio de peregrinação do usuário.

Para valorizar a competência comunitária, a competência daquele médico de família e também a coordenação do cuidado junto com a equipe. E o papel dele, em si mesmo, que é ser o responsável por aquela unidade, acompanhar o desempenho clínico daquela unidade. (Gestor 4).

O fato de a ideia de regulação descentralizada ter respondido à demanda de aumento da capacidade regulatória e de aposta na maior coordenação do cuidado pela APS contribui para que o modelo pareça, muitas vezes, nas entrevistas, uma escolha óbvia, como se outras alternativas não tivessem sido cogitadas ou fossem impossíveis. Entretanto, isso parece esconder um processo que foi, na verdade, produto de disputas entre diversos atores e de análise das outras experiências locais, conforme indicam os dados coletados em campo.

Por exemplo, a ideia de investir na central ou em pontos regionais de regulação foi ignorada - propositalmente ou não - a partir de perspectivas políticas e até mesmo econômicas dos atores. Entre as justificativas de alguns entrevistados que inviabilizariam uma central mais robusta, destacam-se uma impossibilidade de se contratar reguladores centrais por dificuldades financeiras municipais e certa incapacidade desse ponto da rede de atender ao volume regulatório do MRJ. Essas ideias colaboram um 'apagamento' da central de regulação nos discursos sobre o processo de regulação.

Entretanto, o argumento de 'dificuldades financeiras' contrasta com grandes investimentos municipais na APS no mesmo período ${ }^{13}$, desconsiderando, também, que a superação de dificuldades operacionais no acesso ambulatorial passa pelos investimentos em outros pontos da rede além do nível primário, como a própria central de regulação.

Ademais, um município de grande porte requereria uma central muito mais robusta (em termos de profissionais e em capacidade de articulação com outros pontos de atenção) ${ }^{15}$ para se obter maior capacidade de execução das atividades demandadas pelo sistema de saúde, ou mesmo arranjos regionalizados de regulação - a exemplo do que acontece em Recife ${ }^{23}$. Esse espaço, inclusive, permanece com funções essenciais à regulação no MRJ até hoje, como a articulação com os prestadores de serviços, a disponibilização de vagas no Sistema de Regulação (Sisreg) para os encaminhamentos pela APS e até mesmo o agendamento de algumas consultas e procedimentos ${ }^{\mathbf{1 4}}$.

\section{Decisão: a prerrogativa de regulação pelas unidades de APS}

O momento de decisão é marcado pela 'oficialização' do modelo descentralizado. Esse processo foi acompanhado de alterações na SMS-RJ - tanto de estruturas organizacionais quanto de suas lideranças - e por um forte discurso para os profissionais em unidades de APS sobre o novo formato: "A regulação descentralizada é ótima para vocês [profissionais da APS], vai resolver a vida de vocês, que hoje está tudo emperrado" (Gestor 1).

Consolidou-se o formato do modelo descentralizado e o uso da regulação para afirmar a APS como porta de acesso às vagas da rede no MRJ. Criaram, por exemplo, regras sobre processo de trabalho, primeiras linhas de cuidado e formas dos fluxos de pacientes (como protocolos de encaminhamentos e caminhos de primeira consulta e retorno).

A escolha dos médicos Responsáveis Técnicos das unidades (RT) para, além das tarefas assistenciais e de governança

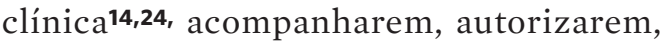
negarem e pedirem revisão dos encaminhamentos feitos pelos outros profissionais da unidade é feita sob diversas justificativas. Esse profissional já recebia bonificação salarial, portanto, segundo um entrevistado, a decisão foi uma maneira de 'formalizar' (sic) o que alguns RT já faziam: avaliar a pertinência clínica dos pedidos de outros reguladores. 
A gente mudou esse processo de regulação, colocando-o sob a responsabilidade do médico responsável técnico por aquela unidade, que é o RT, que já ganhava $20 \%$ a mais por essa responsabilidade. [...] Ele comeccou a gerenciar, ter a senha de regulador para garantir que os pacientes que fossem regulados tivessem de fato um melhor encaminhamento e fossem marcados considerando todos esses aspectos e atributos da atenção primária. (Gestor 4).

Por outro lado, justificou-se a escolha a partir do fato de a posição ser ocupada idealmente pelo médico mais qualificado da unidade, que advogaria pelo paciente com maior capacidade de gerenciar os encaminhamentos conforme as circunstâncias clínicas, do usuário e territoriais. "Então, optou-se pelo médico mais qualificado da unidade. Ninguém melhor do que aquele médico para advogar pelo seu paciente. Era esse o discurso" (Gestor 2).

É importante ressaltar que essa justificativa não se concretiza no cotidiano de toda APS. Aparentemente, em algumas unidades, o RT é realmente o profissional mais capacitado, e sua atuação resulta em maior pertinência clínica dos encaminhamentos, o que não é a realidade de muitos lugares, onde nem sempre o cargo é ocupado pelo 'mais qualificado que advoga por seus pacientes'.

Nesse primeiro momento, também foi definido que os reguladores poderiam regular todos os procedimentos, inclusive os mais complexos e raros na rede municipal, o que, posteriormente, foi modificado, com centralização de alguns procedimentos. "Em 2012, foi tudo ou nada. Então foi tudo ofertado, $e$ qualquer regulador de qualquer nível poderia agendar qualquer coisa" (Gestor 1 ).

Sobre a distribuição da oferta ambulatorial para a APS, destacou-se a decisão sobre implementação ou não de cotas parametrizadas das vagas entre unidades ou áreas programáticas do município. A gestão do Rio de Janeiro optou por processo de trabalho em que cada RT pudesse agendar ilimitadamente por sua unidade de APS, sob argumento de que um formato com reserva de vagas pré-estabelecidas geraria sobra de vagas caso o RT não agendasse todos os exames e consultas disponibilizados para sua unidade.

Embora isso tenha permitido grande volume de agendamentos, constata-se na descentralização uma ausência de ideias ou ações para melhor distribuição e funcionamento territorial dos serviços especializados ambulatoriais pela cidade - por exemplo, como ocorreu na experiência de Belo Horizonte, com a criação dos Centros de Especialidades Médicas para regionalização da atenção especializada"1. É importante reconhecer, no MRJ, a existência dos NIR (Núcleo Interno de Regulação), que são estruturas regionais que participam da regulação, oferecendo mediação e suporte às unidades de APS, mas que não têm poder sobre a programação/distribuição de vagas.

A falta de regionalização da oferta acarreta problemas no Rio de Janeiro, uma cidade de grande extensão territorial e população, uma vez que nem sempre é simples acessar os serviços por questões relacionadas à distância, ao transporte público, à violência urbana ou até mesmo à pequena comunicação entre os níveis. Segundo Canonicci25, integrar níveis de atenção é fundamental para a APS construir coordenação do cuidado e longitudinalidade ao se consolidarem meios de ordenamento da rede.

Problemas de difícil manejo/resolução surgiram na experiência descentralizadora do Rio de Janeiro de não adoção de cotas parametrizadas por unidade na regulação descentralizada, como será visto a seguir. Destaca-se o contraste entre dois entrevistados, pois, enquanto um opina que o modelo foi construído com poucas falhas, outro considera certo 'atropelamento' em algumas decisões, caracterizadas por oportunismo e pequeno espaço de diálogo para alguns atores, o que teria reverberado imediatamente durante a implantação do modelo. Isso, em parte, relaciona-se com o limite comum na previsão de possíveis consequências, imediatas ou de longo prazo, na fase 'decisão' 19 . 


\section{Implementação: a regulação afetada pelas disparidades da APS e da arquitetura regulatória}

Implementar uma política envolve articular diferentes esferas gerenciais do serviço público para a mobilização de agentes implementadores, além de recursos materiais e imateriais ${ }^{19}$. Na experiência da descentralização, isso se concretizou em mudanças no processo de trabalho e no espaço físico das unidades básicas, além das criações de novos cargos relacionados à regulação, entre outras, chamando atenção para a dificuldade de adaptação de alguns profissionais: "se faziam alguns seminários extremamente lotados porque as pessoas tinham que entender como isso funcionava. Poucos entendiam como funcionava" (Gestor 3).

A descentralização no MRJ se caracteriza mais como processo ‘top-down', já que a organização central controlava a maior parte dos fatores relacionados à implementação, e os gestores centrais e profissionais aliados/ próximos da SMS-RJ aparecem como protagonistas em um processo pouco marcado pela participação de outros profissionais da APS na construção do modelo descentralizado.

Um exemplo disso nas entrevistas eram as articulações relacionadas à gestão da fila de espera por consultas e procedimentos no município, iniciadas antes mesmo da oficialização do novo formato regulatório. As ações municipais incluíram mutirões de regulação: dias (geralmente finais de semana) em que gestores e reguladores locais mais próximos deles faziam o que alguns entrevistados definiram como 'a limpeza da fila'.

Ademais, registra-se nas entrevistas que diferentes graus de envolvimento dos grupos profissionais na implantação do modelo foram responsáveis por visões variadas sobre suas consequências. Isso é exemplificado com o problema de sobrecarga dos RT: gestores relatam o cotidiano de trabalhar dentro e fora das unidades, muitas vezes, além do horário de expediente e até mesmo durante a madrugada, a fim de se atender à quantidade de encaminhamentos das equipes locais.

Ainda assim, um grupo de RT, principalmente os que trabalhavam nas unidades maiores ou perto dos gestores municipais, aparentemente não se queixava tanto da elevada exigência de dedicação profissional que surgiu com o novo processo de trabalho. Dessa maneira, segundo um dos gestores, era possível dividir os responsáveis técnicos em dois grupos: a maioria que entendia a regulação descentralizada como um fardo; e uma minoria que aparentemente tornou o sucesso da regulação descentralizada até mesmo um projeto pessoal - o 'desejo de fazer dar certo':

Então, existia pela parte principalmente de alguns atores, de algumas pessoas-chave, que eram os $R T$ das clínicas maiores, as clínicas que tinham residência médica, de querer fazer o negócio dar certo. E uma parte maior, de outros médicos que não estavam tão próximos da secretaria, que não estavam tão próximos de programas de formação, que muitas vezes não tinham nem qualificação necessária para ser RT, estavam ali porque tem que ter. [...] Então, para esses caras, e eu pude perceber isso quando virei coordenador de área, a tarefa da regulação era um fardo. (Gestor 1).

Esse cenário produz iniquidades no acesso dos usuários que persistem até os dias atuais, na medida em que médicos reguladores que acessam mais o Sisreg, inclusive fora do horário de trabalho, conseguem mais vagas para os pacientes de suas unidades.

É porque a regulação, hoje, da forma que ela funciona descentralizada, ela não é igual para todo mundo. O cara que está numa unidade onde o médico regulador não entra no sistema não tem o mesmo direito de acesso do que outro que é de uma unidade onde tem um 'tarado' que fica o dia inteiro de olho na tela. (Gestor 2).

Dessa maneira, o modelo descentralizado promoveu competição pelas vagas, sobrecarga 
dos RT e não impediu que usuários com menor gravidade consigam cuidados especializados antes de usuários com maiores complicações clínicas de outras unidades, se o profissional responsável por seu agendamento acessar constantemente o sistema de regulação. Isso se opõe ao efeito motivador das atividades regulatórias, que é esperado quando elas são entendidas pelos profissionais como ferramentas para facilitação e efetivação da equidade ${ }^{26}$. Alguns entrevistados reconhecem o problema:

A regulação descentralizada ela é necessária, mas no modelo, no molde que ela se encontra, ela ainda é muito desigual, muito injusta. É lei da oferta e da procura do mais rápido na frente do computador na hora que sai a vaga. (Gestor 5).

Visando a reduzir esses problemas, o horário permitido para agendamento foi limitado das $7 \mathrm{~h}$ às $22 \mathrm{~h}$, impedindo a regulação durante a madrugada, mas não evitando o trabalho fora do expediente. Além disso, foi recomendado que os reguladores reservassem 4 horas semanais para atividades regulatórias $^{27}$, mas gestores apontam falta de adesão dos médicos, que alegam inviabilidade, devido às constantes demandas assistenciais durante o dia de trabalho.

Portanto, tais efeitos não foram enfrentados de maneira efetiva, o que é justificado por alguns gestores por uma limitação do software utilizado, que não permite regionalização da oferta. No entanto, Spedo et al. ${ }^{5}$ mostram, a partir da experiência de São Paulo, que, mais importante do que a capacidade informacional de operação do sistema, seu funcionamento depende, principalmente, do envolvimento adequado de diferentes atores na resolução de problemas. Nesse sentido, é fundamental se pensar em outros arranjos possíveis para a regulação do Rio de Janeiro, inclusive usando o próprio Sisreg, ou mobilizar outras alternativas - o que não pode ser descartado, principalmente em um município que tanto investiu na APS (como em compra de prontuários eletrônicos).

\section{Avaliação: ganhos, perdas e a necessidade de revisitar a descentralização}

O modelo descentralizado sofreu, desde 2012, poucas reformulações significativas para a diminuição do fardo dos reguladores ou outras dificuldades. Isso parece relacionado ao fato de que, diferentemente de outras experiências ${ }^{5}$, houve pequena ênfase durante a experiência descentralizadora no MRJ na etapa de 'avaliações': uso de critérios para subsidiarem a tomada de novas decisões ou os resultados de uma política'19,20.

A partir do estudo, é possível notar repercussões positivas do modelo descentralizado. Entre os sucessos mais comentados, destacam-se a redução das filas de espera, maior pertinência clínica dos encaminhamentos, fortalecimento da coordenação do cuidado e mais protagonismo da APS no acesso ambulatorial.

A melhoria no acesso à atenção especializada é apontada como grande ganho da regulação descentralizada por gestores do nível central e local, oportunizando a regulação de vagas de maneira mais rápida quando comparada à regulação apenas nas centrais de regulação. Atualmente, em alguns casos, conseguem um agendamento até no mesmo momento do atendimento na unidade de APS, o que demonstra maior agilidade e organização do processo.

Eu acho que foi um momento importantíssimo para a história do SUS no Brasil. Muitas pessoas foram beneficiadas pela descentralização. [...] Houve uma ampliação do acesso gigante e houve um empoderamento do processo de atenção primária como coordenadora do cuidado. Se ele é o centro coordenador do cuidado, é ela que tem que referenciar o paciente. (Gestor 3).

Nesse sentido, há uma valorização da descentralização por parte de gestores, atribuindo-se a ela um grande avanço no acesso à atenção especializada pelo aumento do número de reguladores. Pinto et al..$^{\mathbf{1 3}}$ apontam que o número de procedimentos, exames e 
consultas agendados no MRJ entre $2011 \mathrm{e}$ 2015 sofreu um aumento de $86 \%$, passando de 790.091 para 1.469.771, o que seria reflexo da descentralização da regulação.

No entanto, é preciso considerar, também, um possível aumento da oferta. Rocha ${ }^{15}$, ao eleger 4 especialidades e compará-las antes e depois da descentralização da regulação (2011 a 2013), verificou um aumento da qualidade da regulação e uma diminuição do tempo de espera, mas a redução da espera foi constatada justamente nas especialidades em que houve, simultaneamente, um aumento da oferta.

Em vez de se configurar como uma consequência direta da descentralização, o aumento da oferta de vagas no Sisreg se relaciona mais ao empenho político e à capacidade de negociação dos gestores na contratualização de serviços. Para Cecílio et al. ${ }^{6}$, o controle de vagas se configura uma disputa entre os diferentes regimes regulatórios, destacando-se no Rio de Janeiro a oposição entre a regulação clientelística (oferecer ao usuário um by-pass aos fluxos normais do acesso a um serviço com intenção principal de se obter seu voto em eleições) e a regulação governamental (a disponibilização equânime, ordenada, oportuna e qualificada da alternativa de assistência mais adequada à necessidade do cidadão por meio de complexos reguladores) ${ }^{28}$.

No contexto de expansão da APS, gerenciar o acesso à atenção especializada aparece muitas vezes associado à justificativa de que a capacidade instalada do MRJ, se disponível no Sisreg para a regulação ambulatorial, zeraria as filas de espera. Para Pinto et al. ${ }^{13}$, pelo menos metade da oferta das esferas estadual e federal seria suficiente para quase eliminar os tempos médios de espera de várias consultas e exames. Cabe destacar, porém, que as filas de agendamento para procedimentos não são obrigatoriamente o critério mais adequado para avaliar a necessidade municipal de algum serviço ambulatorial ou especialidade/exame, pois 'zerar as filas' não significa, forçosamente, oferecer atendimento a todos os usuários da cidade que precisam de algum cuidado especializado, além de existirem parâmetros mais consistentes para definir a necessidade de atenção especializada municipal.

Ademais, não há exatamente uma simultaneidade cronológica entre o novo formato de acesso e o crescimento do número de serviços no sistema, ainda que, obviamente, o aumento do número de horas médicas na regulação contribua para um maior número de procedimentos regulados e agendados. Entre 2009 e 2011, as vagas conquistadas pela iniciativa da SMS-RJ ainda eram gerenciadas apenas pela central de regulação, e a descentralização ainda estava em processo de formulação, sendo implementada apenas em meados de 2012. Desse modo, a regulação ambulatorial pela APS não é a causa direta do aumento de consultas e exames sob controle municipal.

A descentralização da regulação também fortaleceu a coordenação do cuidado pela APS quando deu o poder de agendamento aos médicos que conhecem os pacientes e estão próximos a eles. Uma regulação realizada pela central tende a ser baseada apenas no conteúdo escrito, o que gera problemas na medida em que há menor diálogo entre o profissional que solicita e o que agenda. Esse problema é atenuado no modelo do Rio de Janeiro, já que o nível básico passou a estar bastante inserido no processo regulatório municipal, ocorrendo mais interações entre solicitante e regulador numa mesma unidade.

Cabe destacar, porém, que, no MRJ, o empoderamento da APS como ordenadora dos fluxos é um processo marcado por eventos e decisões insuficientes para efetivar a coordenação do cuidado, conforme sugerem as falas dos entrevistados. Parte disso se deve ao fato de o processo descentralizador ser marcado por aparente fixação dos discursos e esforços na APS, de modo que não se identificou forte debate sobre o lugar reservado ao nível especializado. A atenção especializada, de certa maneira, é colocada em papéis, funções e entradas pouco definidos/marcados/ posicionados com relação à rede, parecendo se resumir a um ofertante das vagas mesmo após a implantação do novo formato regulatório. 
Ao que parece, não ocorreram mudanças significativas em sua conformação organizacional (como parte da rede), nem no modelo de atenção ou na lógica de trabalho/cuidado internos aos cuidados ambulatoriais, que também é (ou deveria ser) um lugar de disputas e reorientações 5,9-11,26. Para Almeida29(246), coordenar envolve "fluxos e contrafluxos de pessoas, produtos e informações e a operação de mecanismos e instrumentos diversos", uma lógica que não se estabelece no município do Rio, pois o nível primário ainda não tem garantida sua comunicação com outros pontos do cuidado. Nesse sentido, há incapacidade em se garantir uma continuidade na atenção e/ou no reconhecimento de problemas que impedem a construção de um cuidado colaborativo pela rede, exemplificado durante o processo de trabalho com o pequeno uso de ferramentas como a contrarreferência na comunicação entre os níveis.

Portanto, esse trabalho aponta que a regulação descentralizada contribui para maior capacidade municipal de organização e controle das vagas se comparado ao modelo anterior do MRJ. No entanto, como pôde ser visto, algumas mudanças apontadas nas entrevistas, embora fortemente influenciadas pela regulação local, não podem ser atribuídas exclusivamente ao novo modelo, o que revela certa confusão - não se sabe se propositalmente ou não - nas narrativas construídas por alguns gestores.

Parte dessas associações parece advir do fato de a descentralização ser pouco acompanhada de avaliações ex-post - ocorridas durante e após a implementação da política -, de modo que, além de consequências negativas imediatas não parecerem ter sofrido intervenções, atribuíram-se à regulação descentralizada méritos que parecem extrapolá-la. A descentralização se posiciona, assim, como parte de um conjunto maior de mudanças.

Cabe indicar, por fim, que é razoável supor, também, que o desinvestimento municipal na APS nos últimos anos possivelmente fragilizou, ainda que indiretamente, o funcionamento da regulação descentralizada, apesar desta ter permanecido com a mesma lógica.

O quadro 3 resume o processo de descentralização da regulação ambulatorial. $\mathrm{O}$ uso do ciclo permite uma distinção didática de atores, discursos, intenções, momentos e repercussões, e possibilita, assim, a

percepção de que existem diferentes momentos no processo de construção de uma política, apontando para a necessidade de se reconhecer as especificidades de cada um destes momentos, possibilitando maior conhecimento e intervenção sobre o processo político ${ }^{19(228)}$.

\footnotetext{
Quadro 3. A iniciativa de regulação ambulatorial descentralizada no município do Rio de Janeiro - Rio de Janeiro, 2021

Reconheci- Aposta por novo formato de regulação em contexto crônico de peregrinação dos pacientes numa mento da rede pouco articulada, uma central de regulação insuficiente para a demanda municipal e uma APS problemática com lugar restrito de solicitante. Simultaneamente, ocorria na cidade um investimento em APS desde e montagem 2009, com grande disputa entre prefeitura e outros atores pelo controle das vagas.

da agenda

Formulação Idealização de um modelo descentralizado que foi atribuído ao fortalecimento da coordenação do da política cuidado pela APS no contexto de sua expansão. A regulação local foi apontada pelos gestores como única alternativa de se aumentar a capacidade regulatória, de modo a atender à demanda municipal, embora o desinvestimento na central de regulação tenha sido uma opção.

Tomada de Iniciaram-se mudanças organizacionais e grande empoderamento político das esferas relacionadas decisão com a APS e o modelo descentralizado, assim como a criação do novo processo de trabalho. Momento marcado pela incrementalidade e pelo oportunismo, de modo que não aparecem muitos diálogos entre gestão central e profissionais da ponta. Isso, juntamente com outros fatores, limitou a capacidade de previsão das repercussões originadas das decisões tomadas.
} 


\begin{tabular}{ll}
\hline Quadro 3. (cont.) & \\
\hline Implemen- & Em meados de 2012, a regulação é descentralizada para a APS, e o período é marcado por grandes \\
tação & mudanças dos espaços e processos de trabalho, além de esforços como 'mutirões de regulação'. \\
& Esbarra-se imediatamente nas diferenças entre unidades e profissionais em cada lugar e também na \\
& sobrecarga do RT. \\
& Uma etapa pouco enfatizada no desenvolvimento da regulação ambulatorial descentralizada do Rio \\
& de Janeiro. É possível atribuir ao novo modelo certo sucesso na redução de filas, no controle dos \\
& fluxos e na mudança de lugar da APS no processo regulatório, embora isso não seja consequência \\
& apenas do acesso descentralizado. As repercussões negativas de acúmulo de funções pelo RT e a \\
& competição por vagas no Sisreg entre as unidades não são alvos de intervenções, de modo que essas \\
& problemáticas se tornam crônicas.
\end{tabular}

Fonte: elaboração própria a partir do ciclo de políticas públicas de Howlett e Ramesh 20 .

\section{Considerações finais}

A análise da descentralização do acesso para a APS no MRJ inspirada no ciclo proposto de Howlett e Ramesh relaciona passado e presente, revelando limites e alcances construídos desde as primeiras ideias de um novo modelo regulatório.

As entrevistas apontam como méritos do acesso ambulatorial, a partir da APS no MRJ, a ampliação tanto da pertinência clínica dos encaminhamentos quanto do acompanhamento pelos profissionais dos fluxos dos usuários. Em contrapartida, alguns ‘sucessos' não são reflexos exclusivos da descentralização (o aumento da capacidade regulatória), e outros parecem não se concretizar plenamente (a coordenação do cuidado pela APS). Ademais, surgem consequências negativas, como exemplificado na sobrecarga dos RT e no 'apagamento' da central de regulação no processo regulatório.

$\mathrm{O}$ modelo permanece promissor, em que pese seus desafios na construção do acesso ambulatorial adequado, em tempo oportuno e integrado à rede de cuidados. A partir da adoção de processos avaliativos, pode-se viabilizar a criação de outros arranjos regulatórios, de maneira a incorporar a equidade ao modelo descentralizado, permitindo novos avanços para usuários, profissionais e instituições do sistema de saúde municipal.

A divisão em fases/ciclos pode provocar uma aparente independência entre movimentos da descentralização, ignorando sua dinamicidade e imprevisibilidade nas diversas disputas. Por outro lado, essa separação revela a necessidade de se reconhecerem as especificidades das etapas para maior conhecimento e intervenção sobre o processo técnico-político.

\section{Colaboradores}

Guabiraba K (0000-0001-7658-8689)*, Gomes G (0000-0001-5608-0183)* e Melo EA (00000001-5881-4849)* e contribuíram igualmente para a elaboração do manuscrito. 


\section{Referências}

1. Brasil. Lei $\mathrm{n}^{\mathrm{o}}$ 8.080, de 19 de setembro de 1990. Dispõe sobre as condições para a promoção, proteção e recuperação da saúde, a organização e o funcionamento dos serviços correspondentes e dá outras providências. Diário Oficial da União. 19 Set 1990.

2. Melo EA, Gomes GG, Carvalho JO. A regulação do acesso à atenção especializada e a Atenção Primária à Saúde nas políticas nacionais do SUS. Physis. 2021; 31(1):e310109.

3. Brasil. Ministério da Saúde. Portaria $n^{\circ} 1.559$, de $1^{\circ}$ de agosto de 2008. Institui a Política Nacional de Regulação do Sistema Único de Saúde - SUS. Diário Oficial da União. 2 Ago 2008.

4. Conill EM, Giovanella L, Almeida PF. Listas de espera em sistemas públicos: da expansão da oferta para um acesso oportuno? Considerações a partir do Sistema Nacional de Saúde espanhol. Ciênc. Saúde Colet. $2011 ; 16(6): 2783-2794$.

5. Spedo SM, Pinto NRS, Tanaka OY. O difícil acesso a serviços de média complexidade do SUS: o caso da cidade de São Paulo, Brasil. Physis. 2010; 20(3):953972.

6. Cecilio LCO, Carapinheiro G, Andreazza R, et al. O agir leigo e o cuidado em saúde: a produção de mapas de cuidado. Cad. Saúde Pública. 2014;30(7):15021514.

7. Brasil. Ministério da Saúde. Portaria no 2.488, de 21 de outubro de 2011. Política Nacional de Atenção Básica. Diário Oficial da União. 22 Out 2011.

8. Brasil. Ministério da Saúde. Portaria $n^{\circ} 2.436$, de 21 de setembro de 2017. Política Nacional de Atenção Básica. Diário Oficial da União. 21 Set 2017.

9. Baduy RS, Feuerwerker LCM, Zucoli M, et al. A regulação assistencial e a produção do cuidado: um arranjo potente para qualificar a atenção. Cad. Saúde Pública. 2011; 27(2):295-304.
10. Silveira MSD, Cazola LHO, Souza AS, et al. Processo regulatório da Estratégia Saúde da Família para a assistência especializada. Saúde debate. 2018; 42(116):63-72.

11. Dias MP. Estratégias de coordenação entre a atenção primária e secundária à saúde no município de Belo Horizonte. [dissertação]. Rio de Janeiro: Escola Nacional de Saúde Pública Sergio Arouca, Fundação Oswaldo Cruz; 2012.

12. Silva JRS. Regulação Assistencial e Atenção Básica em algumas experiências estudadas no Brasil. [monografia]. Rio de Janeiro: Escola Nacional de Saúde Pública Sergio Arouca, Fundação Oswaldo Cruz; 2017.

13. Pinto LF, Soranz D, Scardua MT, et al. A regulação municipal ambulatorial de serviços do Sistema Único de Saúde no Rio de Janeiro: avanços, limites e desafios. Ciênc. Saúde Colet. 2017; 22(4):1257-1267.

14. Gonçalves JB. Análise dos encaminhamentos para primeira consulta em oftalmologia geral a partir de dados do Sistema de Regulação (SISREG III) no município do Rio de Janeiro em 2017. [dissertação]. Rio de Janeiro: Escola Nacional de Saúde Pública Sergio Arouca, Fundação Oswaldo Cruz; 2019, 215 p.

15. Rocha AP. Regulação Assistencial Ambulatorial no município do Rio de Janeiro - RJ: efeitos da inserção da APS na Regulação. [dissertação]. Rio de Janeiro: Escola Nacional de Saúde Pública Sergio Arouca, Fundação Oswaldo Cruz; 2015. 95 p.

16. Instituto Brasileiro de Geografia e Estatística. Cidade do Rio de Janeiro: População Estimada; 2019. [acesso em 2020 dez 22]. Disponível em: https://cidades. ibge.gov.br/brasil/rj/rio-de-janeiro/panorama.

17. Rio de Janeiro. Plano Municipal de Saúde do Rio de Janeiro: PMS 2014-2017. Rio de Janeiro: Secretaria Municipal de Saúde; 2013.

18. Gomes RA. Análise de dados em pesquisa qualitativa. In: MCS Minayo, organizadora. Pesquisa Social. 
Teoria, método e criatividade. 18. ed. Petrópolis: Vozes; 2001.

19. Mattos RA, Baptista TWF, organizadores. Caminhos para análise das políticas de saúde. Porto Alegre: Rede Unida; 2015.

20. Howlett M, Ramesh M. Studying Public Policy. Canada: Oxford University Press; 1995.

21. Kingdon J. Agendas, alternatives and public choices. Boston: Little Brown; 1984.

22. Brasil. Ministério da Saúde. Portaria ${ }^{\circ} 1.792$, de 22 de agosto de 2012. Institui incentivo financeiro de custeio destinado às Centrais de Regulação organizadas no âmbito do Sistema Único de Saúde (SUS). Diário Oficial da União. 23 Ago 2012.

23. Albuquerque MSV, Lima LP, Costa AM, et al. Regulação assistencial no Recife: possibilidades e limites na promoção do acesso. Saúde soc. 2013; 22(1):223236.

24. Rio de Janeiro. SISREG - Protocolo para o Regulador. Protocolo Clínico de Critérios para Regulação de Vagas Ambulatoriais. Rio de Janeiro: Secretaria Municipal de Saúde; 2015.
25. Canonici EL. Modelos de unidades e serviços para organização da atenção ambulatorial especializada em Sistemas Regionais de Atenção à Saúde. São Paulo: Proadi-Sus; 2014.

26. Peiter CC, Lanzoni GMM, Oliveira WF. Regulação em saúde e promoção da equidade: o Sistema Nacional de Regulação e o acesso à assistência em um município de grande porte. Saúde debate. 2016; 40(111):6373.

27. Rio de Janeiro. Prefeitura Municipal do Rio de Janeiro. SMS-RJ. Ofício Circular S/SUBPAV/SAP n ${ }^{\circ}$ 09/2018. Diário Oficial do Estado do Rio de Janeiro. 23 Maio 2018.

28. Brasil. Ministério da Saúde. Portaria $\mathrm{MS} / \mathrm{GM} \mathrm{n}^{\circ} 373$, de 27 de fevereiro de 2002. Norma Operacional de Assistência à Saúde/NOAS-SUS. Diário Oficial da União. 27 Fev 2002.

29. Almeida PF, Medina MG, Fausto MCR, et al. Coordenação do cuidado e Atenção Primária à Saúde no Sistema Único de Saúde. Saúde debate. 2018; 42(esp1):244-260

Recebido em 18/06/2021

Aprovado em 24/10/2021

Conflito de interesses: inexistente

Suporte financeiro: não houve 